\title{
Maternal perceptions of 5-year-old child weight status and factors influencing perceptions: Findings from the ROLO Kids Study
}

\author{
Anna Gouldson, Aisling Geraghty, Eileen O’Brien, Mary Horan, Jean Donnelly, \\ Elizabeth Larkin and Fionnula McAuliffe \\ UCD Perinatal Research Centre, 65-66 Mount Street Lower, Dublin 2, Ireland
}

\section{Abstract}

Introduction: Obesity is a global condition affecting both adults and children. Mothers play an important role in identifying problems with their child's weight. However, studies are limited on the accuracy of maternal perceptions of her child's weight status in early childhood. The aim of this study was to look at maternal perceptions of her child's weight status in 5-year-old children and to investigate factors influencing this.

Materials and Methods: 339 mother and child pairs were followed up from the ROLO Kids study in the National Maternity Hospital, Dublin, Ireland. Height and weight of the mother and the child were measured, and BMI was calculated. Demographic details were collected and questionnaires asked mothers to assign their child to a weight category and to give the reason they put their child in this particular category. KAPPA analysis and student $t$ tests were used to analyse the data.

Results: In this cohort over $75 \%$ of the children were normal weight, $14.7 \%$ were in the overweight category, and $8.5 \%$ in the obese category. The KAPPA score for maternal-perceived category and actual weight status was 0.037 which is a low level of agreement. Mothers were more likely to underestimate their child's weight, with $28.5 \%$ underestimating the appropriate weight category for their child compared with $2.4 \%$ who overestimated. Maternal age, education, and child sex did not influence accurate weight assessments; however, mothers with a higher BMI were more likely to inaccurately assess their child's weight $(\mathrm{P}<0.05)$.

Discussion: Maternal perception of their child's weight status is not an accurate indication of child weight, particularly for mothers with a raised BMI. This research highlights the important role of health care providers in assessing child weight status, rather than relying on parental reports alone, in order to appropriately intervene to reduce the incidence of childhood obesity.

\section{Conflict of Interest}

There is no conflict of interest 